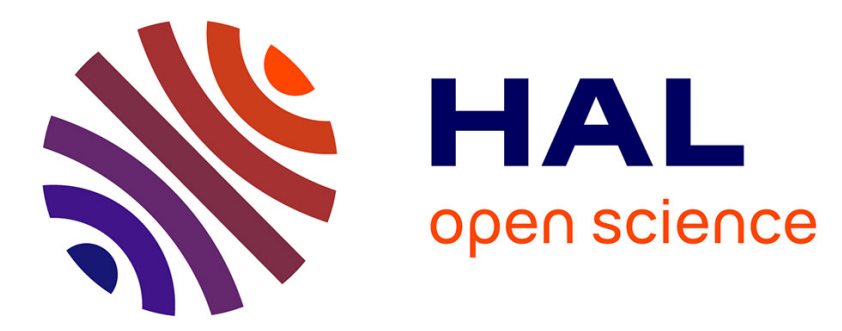

\title{
3D segmentation of abdominal aorta from CT-scan and MR images
}

Anthony A. Duquette, Pierre-Marc Jodoin, Olivier Bouchot, Alain Lalande

\section{To cite this version:}

Anthony A. Duquette, Pierre-Marc Jodoin, Olivier Bouchot, Alain Lalande. 3D segmentation of abdominal aorta from CT-scan and MR images. Computerized Medical Imaging and Graphics, 2012, 36, pp.294-303. hal-00726535

\section{HAL Id: hal-00726535 \\ https://hal.science/hal-00726535}

Submitted on 31 Aug 2012

HAL is a multi-disciplinary open access archive for the deposit and dissemination of scientific research documents, whether they are published or not. The documents may come from teaching and research institutions in France or abroad, or from public or private research centers.
L'archive ouverte pluridisciplinaire HAL, est destinée au dépôt et à la diffusion de documents scientifiques de niveau recherche, publiés ou non, émanant des établissements d'enseignement et de recherche français ou étrangers, des laboratoires publics ou privés. 


\title{
3D Segmentation of Abdominal Aorta from CT-Scan and MR Images
}

Anthony Adam Duquette ${ }^{\mathrm{a}}$, Pierre-Marc Jodoin ${ }^{\mathrm{a}}$, Olivier Bouchot ${ }^{\mathrm{b}}$, Alain Lalande ${ }^{\mathrm{b}}$

a) MOIVRE research center 2500 boulevard de l'Université, Sherbrooke, Québec, Canada J1K 2R1

b) LE2I, UMR CNRS 5158, Faculté de Médecine, Université de Bourgogne, BP 87900, 21079 Dijon Cedex, France

\begin{abstract}
We designed a generic method for segmenting the aneurismal sac of an abdominal aortic aneurysm (AAA) both from multi-slice MR and CT-scan examinations. It is a semi-automatic method requiring little human intervention and based on graph cut theory is proposed to segment lumen interface and aortic wall of AAAs. Our segmentation method works independently on MRI and CT-scan volumes and has been tested on a 44 patient dataset and 10 synthetic images. Segmentation and maximum diameter estimation were compared to manual tracing from 4 experts. An inter-observer study was performed in order to measure the variability range of a human observer. Based on three metrics (the maximum aortic diameter, the volume overlap and the Hausdorff distance) the variability of the results obtained by our method is shown to be similar to that of a human operator, both for the lumen interface and the aortic wall. As will be shown, the average distance obtained with our method is less than one standard deviation away from each expert, both for healthy subjects and for patients with AAA. Our semi-automatic method provides reliable contours of the abdominal aorta from CT-scan or MRI, allowing rapid and reproducible evaluations of AAA.
\end{abstract}

\section{Keywords}

AAA segmentation, MRI, CT scan, Graph Cut, Volume segmentation 


\subsection{Introduction}

The incidence of Abdominal Aortic Aneurysms (AAA) increases with age. The gravity of this disease is due to the growth of the aorta with potential risk of rupture causing a fatal retroperitoneal hemorrhage. In this way, accurate and reproducible measurement of the AAA is a key issue in cardio-vascular surgery. Although abdominal ultrasonography is perhaps the most practical way to screen for aneurysms, contrast-enhanced Computed Tomography (CT) scanning is the preferred imaging modalities in clinical practice. CT-scan images are well adapted to define aortic anatomy, and accurately detect and size aortic aneurysm [1]. As for MRI (Magnetic Resonance Imaging), it offers a non-invasive and yet reliable method for measuring the abdominal aorta. The use of Steady State Free Precession (SSFP) sequence provides a good contrast between the blood, the thrombus (if present) and the aortic wall.

The criteria used for taking the decision to treat an AAA are the maximum diameter (50-55 mm), the volume, and the evolution in time of the aneurysm [2-5]. But measuring the size of a windy aorta based on $2 \mathrm{D}$ images only is error prone. A more precise way of doing so is by measuring the aneurysm on a 3D version of the aorta. Unfortunately, the $3 \mathrm{D}$ reconstruction of the lumen and the wall still needs to be done by hand, a time-consuming procedure infeasible in clinical practice. The use of an automatic or semi-automatic segmentation procedure is thus the only valid option.

Aortic segmentation calls for local segmentation methods, i.e. segmentation methods that can isolate an organ from the rest of the body. On that matter, active contour methods, often called snakes, are certainly the first solutions one might consider [6-9]. One of the main advantages of active contour methods is that it allows for picking out a desired object in an image containing a collection of objects with similar attributes. Unfortunately, snakes are often cumbersome to implement, computationally demanding [10], and can lead to instability (especially those relying on EulerLagrange equations [11]).

Other segmentation methods are conceivable such as flood-fill [12], graph-cut-based methods [12-14], grow cut [15], and watershed [16] to name a few. Unfortunately, as shown in figure 2, these methods often leak when the aorta is pressed against an organ whose grayscale is similar. This can be explained by the fact that these segmentation methods are generic and not well adapted to aortic segmentation. Also, these methods are tone-based and thus assume that the grayscale is uniform inside and outside the contour which is not the case with aortic images. Although similar methods have been used for blood-vessel segmentation [17-21], most of time they were validated on welldefined blood vessels void of a thrombus and for only one modality (MRI or CT-scan). A simple solution to leaking is to initialize the method with a trimap [22-24]. A trimap is a 3-class label field which forces the segmentation to stay within a limited area. However, having to enter a trimap on each image of the volume is time consuming. Although, one could only initialize a subset of images (as is the case for our method), most of the methods end up leaking in the non-initialized images. The inaccurate segmentation results reported in figure 2 are typical of what happens in images void of a trimap initialization.

Another classical solution to leaking is shape priors. Shape priors are used to restrict the end segmentation to some pre-defined shape. A huge effort has been put in adding shape priors to level sets methods [25-30]. Most of these works implement a combination of two functionals: one about the segmentation (it includes image and curve) and one about the shape difference. The segmenting curve then evolves according to two competing forces: 1) the force of the image, and 2) the force exerted by the estimated shape. In general, these methods use either a geodesic edge-based approach [25,31] or a region-based approach [26-28]. Although impressive results are reported in level-set papers, level-set methods with shape priors are not void of drawbacks. They are often slow, do not always generalize well to 3D, and are difficult to re-implement. Furthermore, several level-set methods $[6,27,28]$ have to register the shape model onto the image which is a challenging and computationally expensive task. 
Recently, several graph cut methods with shape prior have been proposed [32-38]. These publications suggest that graph-cut methods are faster and more prone to reach the global minima than level-sets [38]. However, as is the case for most level-set methods, shape-constrained graph-cut methods are somewhat restrictive on the shape $[39,43]$ thus leading to underfitting as shown in figure 2. Other methods $[33,34,38]$ propose a generic shape prior which do not impose the shape of a specific object class. Our experiments reveal that these types of methods are somehow well adapted to aortic segmentation (cf the two right-most images in figure 2). However, these methods are not perfect and often over estimate the aortic wall.

Recently, a graph-cut method [39] has been proposed to segment the aorta's wall provided that the lumen has been pre-segmented. Unfortunately, the method has been designed to work only on CT-Scan volumes with a small slice thickness (less than $1 \mathrm{~mm}$ ). It is thus unclear how the method behaves on MRIs and on volumes with a large slice thickness (up to $6 \mathrm{~mm}$ ).

Segmenting diseased aortas (that is the lumen and the aortic wall) on MRI and CT-scan images is a challenging task on many aspects. The three most glaring problems one has to deal with are the following:

1) By their very nature, MRI and CT-scan picture organs with different grayscales. As shown in figure 1, the lumen appears in bright white in the CT-scan but in midtones in the MRI with SSFP sequence. Also, since the thrombus appears in midtones, the gradient between the lumen and the thrombus is significantly different in these images. The background is also different in both modalities: dark in the CT-scan and mid gray in the MRI. As a result, the aortic wall is only visible in MR Images. Moreover, the images obtained with a black blood MRI sequence (such as T1 spin echo sequence) provide unstable signals through the image. For these reasons, a generic method working independently on MRI and CT-scans volumes cannot rely on tones to differentiate the lumen, the thrombus, the aortic wall, and the background.

2) The aorta is often located next to another organ whose grayscale is the same. As shown in figure 1, the thrombus in the first CT-scan is pressed against the vena cava whose greyscales are identical. In a similar way, the aortic wall in the MRI touches the black spinal column and thus looks as if they were connected. As a result, a number of methods end up leaking in the surrounding organs.

3) The shape and the position of an aorta (be it the lumen or the thrombus) varies greatly from one patient to another. As shown in figure 1, the lumen is elliptic in the MRI but has a peanut shape in the CT-scans (this often happens when a tortuous aorta is pictured in axial images). Eccentric lumens and thromboses are common due to the windy shape of some aortas and the turbulences of the blood flow. Furthermore, the variability between AAA makes the problem even more difficult as the shape and position of the aneurysm is unpredictable.

In this paper, we propose a 3D aortic segmentation method which requires little human intervention and works independently on MRI (with SSFP sequence) and CT-scan volumes. Our method (1) works on edges, (2) does not leak, and (3) recovers a smooth 3D surface without a shape prior. Our solution is thus robust to the three problems just mentioned while requiring little (but intuitive) human intervention. Our method also allows for simple touch-ups in pathological areas. We compared our method to 4 imaging experts who manually segmented the lumen and the aortic wall of 10 synthetic images, 20 axial series of MR, and 24 contrast-enhanced CT image series. 


\subsection{Material and Methods}

\subsection{Study Population and Image acquisition}

Our database contains 35 patients with an AAA (AAA+) and 9 patients without an AAA (AAA-). The table 1 summarizes the study population and the image acquisition parameters. The choice of various image resolutions was intended in order to evaluate our method on a maximum number of plausible set-ups. The MR images were acquired using a free breathing ECG-gated SSFP sequence providing white blood images in an axial orientation. Every image volume covers the abdominal aorta from the renal arteries down to the iliac arteries. The study was conducted in accordance with the ethical standards of the World Medical Association and with the recommendations of the local ethics committee and the French Ministry of Health, and informed consent was obtained from each patient.

\subsection{Metrics}

Methods such as ours are usually validated with respect to ground truths provided by a human observer [17,18]. However, hand-selected results vary from one expert to another, thus leading to biased error estimations. In this paper, we led an inter-observer study to measure the variability between experts. The goal is to show that our method produces results within the human variability range, i.e. that our method is statistically as accurate as an expert can be. Our study involves four human operators, all medical imaging experts. The medical experts involved in this study are people working every day with abdominal tomographic images. This study is based on three metrics used to measure the distance between segmented aortas i.e.:

1- The volume overlap (VO) [40];

2- The Hausdorff distance (HD) [41];

3- The maximum diameter distance (MDD).

Considering that a segmented volume contains voxels classified as being "inside" or "outside" the contour, VO takes the number of voxels labelled as being "inside" in both volumes divided by the total number of voxels labelled as being "inside". Roughly speaking, VO measures how much two volumes overlap in percentage. This metric is also known as the Jaccard index. As for the HD, it is the maximum distance of a segmented volume to the nearest point in the other segmented volume [41]. According to HD, two segmented volumes A and B are close if every point inside the contour $\mathrm{A}$ is close to some point inside the contour $\mathrm{B}$. HD can be seen as the maximum deviation in millimetres between two segmented volumes. The third metric takes the difference between two maximum diameters. For each patient, the maximum diameter is manually entered by the experts but automatically computed by our method based on the 3D shape it estimated.

\subsection{Synthetic images}

Our method has also been validated on synthetic images. One image contains a typical MR image with more or less Rician noise [42]. The other represents a typical CT image with more or less Gaussian noise (figure 3). The grayscale ratio between the different regions in these images is similar to that of real MR and CT images. Both images contain a central zone corresponding to the lumen surrounded by a disc representing the thrombus. In the MRI, the thrombus is surrounded by a thin layer corresponding to the aortic wall. A white area is also added representing the vena cava in the MRI. In both images, a blob has been added below the aorta to picture the back muscle. Note that the small white grey areas at the periphery of the thrombus in the CT image represent 
calcifications. The reason for using synthetic images is to compare (with the help of a ground truth) contours provided by our method to those obtained by manual tracing. Manual tracing was performed independently by an experienced observer on each image.

\subsection{Segmentation Method}

\subsubsection{Graph Cut}

Our segmentation method is inspired by Boykov and Jolly's work [13] which we modified to meet the needs of aortic segmentation. Graph-cut methods use a weighted graph $G=\{\Gamma, \Delta\}$ made of nodes $\Gamma$ interconnected by undirected edges $\Delta$. Each edge $e \in \Delta$ connects two nodes $a, b \in \Gamma$ and is assigned a capacity $w_{e} \geq 0$. A capacity $w_{e}$ indicates the amount of information (or flow) that can circulate between $a$ and $b$. The graph also contains 2 terminal nodes called the source $s$ and the sink $t$ [43].

A concept pivotal to graph cut is the notion of "cut". By nature, graph-cut is a two-class segmentation method which divides (or cuts) the graph in two sets of nodes. Given a graph $G$, a cut $c=(S, T, U)$ separates the node set $\Gamma$ into two subsets, namely $S$ and $T$. To be valid, $S$ and $T$ need to satisfy the following conditions: $S \cap T=\varnothing, S \cup T=\Gamma$, and $s \in S, t \in T$. As for $U$, it contains every edge $e \in \Delta$ that had been cut to split $\Gamma$ into $S$ and $T$. The capacity of a cut is the sum of all edges contained in $U$, i.e.

$$
E(c)=\sum_{e \in U} w_{e}
$$

The goal of graph cut is to find the minimum cut, i.e. the cut $\hat{c}$ whose capacity is minimal

$$
\hat{c}=\arg \min _{c} E(c) .
$$

In our application, the nodes in $S$ are those sitting inside the segmented area and those in $T$ outside the segmentation. Finding the minimum cut of a graph is a known optimization problem for which a number of solutions have been proposed $[44,45]$. In this paper, an alpha-expansion approach is used [46]. For more details on graph cut, please refer to $[13,43,46]$.

\subsubsection{Our Method}

\section{Building the Graph}

The to-be-segmented volume (be it a CTscan or an MRI volume) is made of $L$ images each containing $\mathrm{N} \times \mathrm{M}$ pixels. The volume is made of voxels whose position $(i, j, l)$ indicates their spatial location $(i, j)$ in the $l^{\text {th }}$ image. When building the graph, each node is associated to a voxel in the volume. In this way, the graph contains $\mathrm{L} \times \mathrm{N} \times \mathrm{M}$ nodes plus a source and a sink. Each node (read voxel) at position $(i, j, l)$ in the volume is connected to its 6 neighbours, i.e. $(i+1, j, l),(i-1, j, l),(i, j+1, l)$, $(i, j-1, l),(i, j, l+1)$, and $(i, j, l-1)$. Note that the source and the sink are not associated to a voxel as they are terminal nodes (see figure 4a). The nodes to which $s$ and $t$ are connected depend on the initialization entered by the user.

\section{Initialization}

As shown in figure $4 \mathrm{~b}$, the user is required to roughly select an area of interest. The area can be the lumen or the aortic wall depending on the expected result. We empirically measured that an 
initialization at every $2 \mathrm{~cm}$ is enough to obtain accurate results (that is one contour every 8 to 20 images for a CT-Scan volume and one every 3 to 5 images for an MRI volume).

Once every contour has been drawn, the volume is divided into 3 regions: the "inside" region (in blue), the "neutral" region (in red and in images with no initialization), and the "outside" region (in green). The nodes (read voxels) within the blue sections are connected to the source with an infinite capacity and those in the green area to the drain with an infinite capacity ${ }^{1}$. In this way, the nodes in the blue and in the green areas will be automatically labelled as being "inside" and "outside" the segmented area (i.e. in the " $S$ " and " $T$ " classes). This is a fundamental property of our method as it prevents the results from leaking outside the red contour.

\section{Edge Capacity}

The nodes in the blue and the green regions being automatically clustered in the " $S$ " and " $T$ " classes their edge capacity has no effect on the end result and thus are assigned a constant value. As for the nodes in the neutral region, there edge capacity is assigned the following value given that $a$ and $b$ are neighbours:

$$
w_{e}=\exp \left(-\frac{\delta_{a b}\left|I_{a}-I_{b}\right|^{\gamma}}{2 \sigma^{2}}\right)
$$

where $I$ is the image volume (MRI or CT-scan) and $I_{a}, I_{b}$ stand for the grayscale at voxel $a$ and $b$. This equation also takes into account the gradient direction using a binary $\delta_{a b}$ function. As will be shown later, $\delta_{a b}$ prevents the method from snapping on the edge of the neighbouring organs. Let us also mention that due to the fact that an aorta has different grayscales in CT and MR images, $w_{e}$ contains gradient information instead of intensity information. This is a key element that makes our method suited to both MR and CT images. According to equation (3), the capacity gets low whenever the inter-voxel gradient $\left|I_{a}-I_{b}\right|$ is large. In this way, the method ends up cutting the volume in areas of strong gradient, i.e. on the nearest edges. A "camera noise" parameter $\sigma$ is incorporated which gives more or less influence to the inter-voxel gradient [13].

One common problem arises when the aortic wall exhibits a very low contrast (see the MRI lumen in figure 1). In that case, the method often cuts through a uniform region and thus underestimates the volume. As a solution, we added a parameter $\gamma$ whose value sharpens the image gradient. This parameter can be seen as gradient-based gamma correction.

Another common problem with generic graph-cut solutions occurs when the aorta is located next to an organ whose edges have a larger gradient than that of the aorta. In that case, the method snaps on the edge of a neighbouring organ (see graph-cut and grow-cut results in figure 2). To reduce this effect, we incorporated a notion of "gradient direction" in the edge capacity. As shown in figure 1, the lumen in the CT-scan and the MRI are brighter than their immediate neighbourhood. The situation is similar for the thrombus in the CT-scan. In these cases, when starting from the center of the aorta, the gradient goes from "a light area" to a "dark area", i.e. $I_{a}>I_{b}$ when $a$ is closer than $b$ to the aorta's center. However, the situation is completely different with the aortic wall in the MRI as $I_{a}<I_{b}$. Consequently, a binary $\delta_{a b}$ function is incorporated whose goal is to select the gradients pointing in the right direction:

\footnotetext{
${ }^{1}$ The nodes in the neutral region are not connected to the source or the drain.
} 


$$
\delta_{a b}=\left\{\begin{array}{c}
1 \text { if }\left(d_{a}<d_{b} \& I_{a}>I_{b}\right) \text { or } \quad\left(d_{a}>d_{b} \& I_{a}<I_{b}\right) \\
0 \text { otherwise }
\end{array}\right.
$$

when segmenting the lumen or the wall in the CT-scan, and

$$
\delta_{a b}=\left\{\begin{array}{c}
1 \text { if } \quad\left(d_{a}<d_{b} \& I_{a}<I_{b}\right) \text { or } \quad\left(d_{a}>d_{b} \& I_{a}>I_{b}\right) \\
0 \text { otherwise }
\end{array}\right.
$$

when segmenting the wall in the MRI volume. In both equations, $d_{a}$ (and $d_{b}$ ) stands for the Euclidean distance between $a$ (and $b$ ) and the center of the aorta. The influence of the $\delta_{a b}$ function is illustrated in figure 5. Given a user selection, the end contour will snap of the lumen or the aortic wall depending on whether equation (4) or (5) is used.

\section{Touch Ups}

Once the graph is built, the minimum cut optimizer is launched. The resulting cut corresponds to the best $3 \mathrm{D}$ volume according to the graph's configuration. Although the result is usually good, it is not void of errors, especially in pathological areas. Fortunately, the user can correct local deviations with a simple touch up tool which changes the region index of badly segmented nodes. This tool forces the selected nodes to be part of the inside, outside, or neutral regions. This locally modifies the graph's configuration. Our experiments reveal that up to 5 images usually need to be touched up for a patient. Given that retouching one image requires 4-5 seconds, the average touch up time for a patient does not exceed 20 seconds. The optimizer can then be re-launched to generate a new 3D volume (cf figure 6).

\subsection{Results}

\subsection{Inter-operator Study}

Our method (and the experts) returns a segmentation map as well as an estimation of the maximum diameter for each patient. Based on these data, our goal is to show that our method is as precise as a human observer can be. In that perspective, the average distance (and standard deviation) is computed between the results returned by an operator (be it our method or the experts) to the ones returned by the other four operators. In this way, if our method is as good as a human operator, than its average distance should be similar to that of the other operators. On the other hand, if our method is consistently worse than the experts, its average distance should be systematically larger. Such strategy for detecting outliers is called "distance-based outlier detection" [47]. The average distance that we compute for each operator $\mathrm{O}_{\mathrm{i}}$ is the following:

$$
\operatorname{dist}\left(O_{i}\right)=\frac{1}{4} \sum_{j=1, j \neq i}^{5}\left(\frac{1}{N} \sum_{p} \operatorname{metric}\left(R_{i}^{p}, R_{j}^{p}\right)\right)
$$

where $N$ is the total number of patients, $R_{i}^{p}, R_{j}^{p}$ the result returned by $O_{i}$ and $O_{j}$ on patient $p$, and "metric" is either VO, HD or MDD. The standard deviation is computed in a similar fashion. Note that to make our evaluation robust to outliers, we computed a trimmed average (and standard deviation) by removing the $10 \%$ outlying patients [48].

We first processed the results obtained on the diseased subjects. As can be seen in tables 2, 3 and 4 , the average distance of our method is always within one standard deviation away from each expert. One should note that the largest distance between our method and an observer according to 
the HD and MDD metrics is below 1 millimetre. Given that the spatial resolution of our CT-scan and MR images is between 0.47 to $0.98 \mathrm{~mm}$ and 1.12 to $1.40 \mathrm{~mm}$ respectively, this corresponds to a maximum average error of less than 1 pixel for the MRI and 2 pixels for the CT-Scan. Such error is barely noticeable by a human eye as can be seen in figures 3 and 7 .

Tables 5, 6 and 7 display the results obtained on healthy volunteers. A similar conclusion can be drawn as the results from our method are statistically close to those produced by the four experts. Here again, our method is within one standard deviation away from every expert. The VO distance for our method ranges between $81 \%$ and $89 \%$ which is approximately what we got for the human experts. As for the Hausdorff and the maximum diameter distances, our method is always less than $1 \mathrm{~mm}$ away from the experts.

In order to validate the fact that there is no statistical difference between the experts and our method, we performed an analysis of variance (ANOVA) with the Levene's test of homogeneity of variance. It is commonly accepted that the null hypothesis is verified when the p-value of the test is above 5\%. For the volume overlap, the Hausdorff distance and the maximum diameter distance (tables 2 to 7), we created 5 lists containing the values returned for each patient by our method and the four experts. For these data, the p-values are widely above $5 \%$ which clearly indicate that there is no significant difference between our method and the human experts (the p-value for the maximum diameter distance is equal to $79 \%$ ). We did those tests with a commercial software (SYSTAT for Windows).

For the maximum diameter distance, we also used the Bland-Altman plot to further evaluate the robustness of our method [49]. We took for each patient (be it healthy or diseased) the average maximum diameter entered by the experts and compared it to the results returned by our method. This led to the scatter plot in figure 8 in which each dot corresponds to a patient. The $\mathrm{X}$ and $\mathrm{Y}$ axis correspond respectively to the average and difference between the experts and our method. The error values ( $\mathrm{Y}$ axis) being clearly smaller than the average value ( $\mathrm{X}$ axis), this indicates a good concordance between our method and the four human operators.

\subsection{Synthetic Images}

The results obtained on synthetic images are shown in tables 8,9 and 10 . These tables contain the volume overlap, the Hausdorff distance and the maximum diameter distance for three different noise level $\sigma$. Overall, our method is very close to the human observer especially when the noise level is low. In the worst cases, our method is respectively $4.6 \%{ }^{2}$ and $2.47 \mathrm{~mm}^{3}$ away from the expert. As for the maximum diameter error, our method is at most $0.9 \mathrm{~mm}$ away from the expert which corresponds roughly to a 1-pixel error. As shown in figure 5, these results are both accurate and similar.

\subsection{Performances}

We executed our program on a $2.0 \mathrm{GHz}$ Intel core 2 duo computer with $3 \mathrm{~Gb}$ of RAM. In order to reduce the processing time and save memory, only the region of interest located around the red initial contour is processed. The size of that region varies between $120 \times 120$ and $350 \times 350$ square pixels. Overall, the graph-cut optimizer takes between 0.95 and 2.25 seconds on the MRI volumes and between 0.96 and 8 seconds on the CT-Scan volumes. The hand selection of the initial contours takes between 10 to 40 seconds depending on the number of slices in the volume. The overall procedure including initialization, segmentation and touch up takes roughly less than one minute. This is fairly small compared to manual segmentation. As a rule of thumb, our experts took between

\footnotetext{
${ }^{2}$ In table $8,99.51 \%$ for expert 1 and $94,91 \%$ for our method.

${ }^{3}$ In table $9,2.0 \mathrm{~mm}$ for expert 1 and $4.47 \mathrm{~mm}$ for our method.
} 
4 and 8 minutes to segment a 20-slice MRI volume and between 7 and 12 minutes for a 40-slice CTscan volume.

\subsection{Discussion}

It is well documented that the rupture of an AAA is uncommon when the aneurysm's diameter is below $5.5 \mathrm{~cm}$ and expanding slowly [2-5]. For that reason, the aneurysm size has always been a key parameter in cardio-vascular surgery. Due to the nature of most AAAs, the correct measurement of the aneurysm calls for a full imaging examination. On that matter, CT scans and MRI have always been the preferred modalities, ultrasounds being inapplicable to correctly determine the size and the extent of the aneurysm. Angiographic techniques provide accurate definition of lumen area, but are ineffective in the localization of the aortic wall, restricting the thrombus evaluation. The strength of the multiplanar reformation (MRP) CT scan and the MRI comes from the richness of their data: a volume made of between 20 and 200 axial tomographic images. Although hand selecting the maximum diameter is fairly straight forward, manually determining the size of the aneurysm (useful in surgical repair planning) remains tedious in clinical practice. This is especially true when the full reconstruction of the lumen and the aortic wall is required.

As a solution, we proposed a semi-automatic graph-cut segmentation method to rebuild in 3D the aortic wall and the lumen interface from the renal arteries down to the iliacs. Our method is effective on CT scan images as well as white blood MR images. As we mentioned, basic graph-cut methods such as Boykov and Jolly's [13] are not well suited to the aortic segmentation problem. We thus proposed a cost function that allows (1) to deal with low-contrast edges caused by the windy nature of the aorta and (2) differentiate the lumen from the aortic wall. Furthermore, our method does not leak when the aorta is pressed against another organ which is a known problem with numerous techniques.

Our method is user-friendly and useful in clinical practice as it measures the maximum diameter of the aneurysm as well as the volume, position and eccentricity of the thrombus. It has been validated on data acquired from 44 subjects, some having an AAA and others not. In order to cover different types of acquisitions, various CT scan and MRI settings have been accounted for. Our database has a variety of images with different spatial resolution and slice thickness. Despite these various settings and the fundamental differences between CT scan and MR images, we showed that our method is on the average as precise as the experts. The comparison with four experts underlined the fact that the average distance between our method and manual drawings is similar to the inter-observer variation. Considering the maximum diameter evaluation, the Bland-Altman plot shows that there is a good concordance between the human experts and our method. The slight 3.2 $\mathrm{mm}$ overestimation in the graph is due to the management of the aortic wall by some human operators. Indeed, some operators selected the inner section of the wall while others selected the outer section of the wall. Since our method always use the outer section of the wall, this led to a light shift in the graph.

Also, although our method relies on a user initialization, this procedure is rapid (a few seconds) and intuitive. Since our method is not void of draw-backs, it sometimes overestimates the aortic wall in the vicinity of the collateral arteries. As a solution, we developed a simple touch up tool allowing for manual interventions in pathological areas. Further improvements of the user interface should reduce user intervention.

Our method is still subject to future methodological improvements that hopefully would lead to a complete automatic procedure. We are also working to extend our method to other MRI protocols than the SSFP sequence. More specifically, we would like to make it work on black blood MR sequences such as T1 spin echo sequences. 
As a conclusion, we proposed a semi-automatic method to segment the lumen and the aortic wall from a stack of axial images covering the abdominal aorta from the renal arteries down to the iliacs. Our solution is generic and allows the management of patient examinations taken from CT or MR imagers. A comparison with manual tracing demonstrates that our automatic segmentation is as precise as an expert. The deployment of our approach in clinical practice would provide cardiologists with key parameters allowing the follow up of patients with AAA.

\subsection{Conflict of Interest Statement}

The authors declare having no conflict of interests as the current project has not been founded nor driven by a private healthcare company or any lobby.
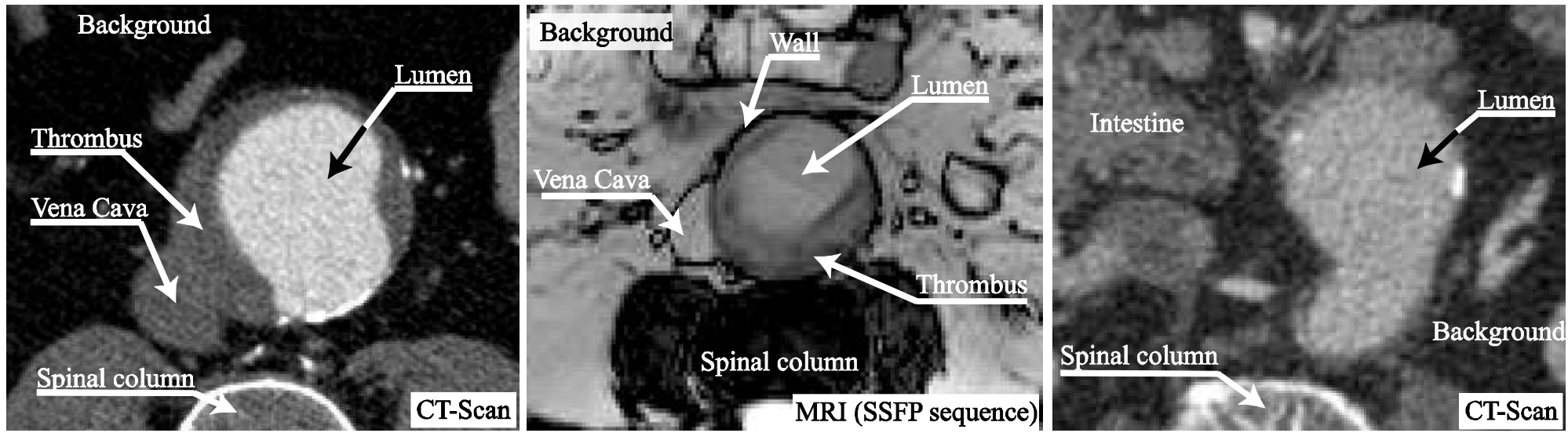

Figure 1 Typical diseased aortas taken from three patients. The first and third images are CT-scan images and the second is an MR image. As can be seen, the shape, the size and the grayscale of the lumen and the thrombus vary significantly from patient to another. These images show diseased aortas with and without a thrombus and with and without the vena cava pressed against the aortic wall. The reader shall notice that the aorta does not always have an ellipsoid shape.
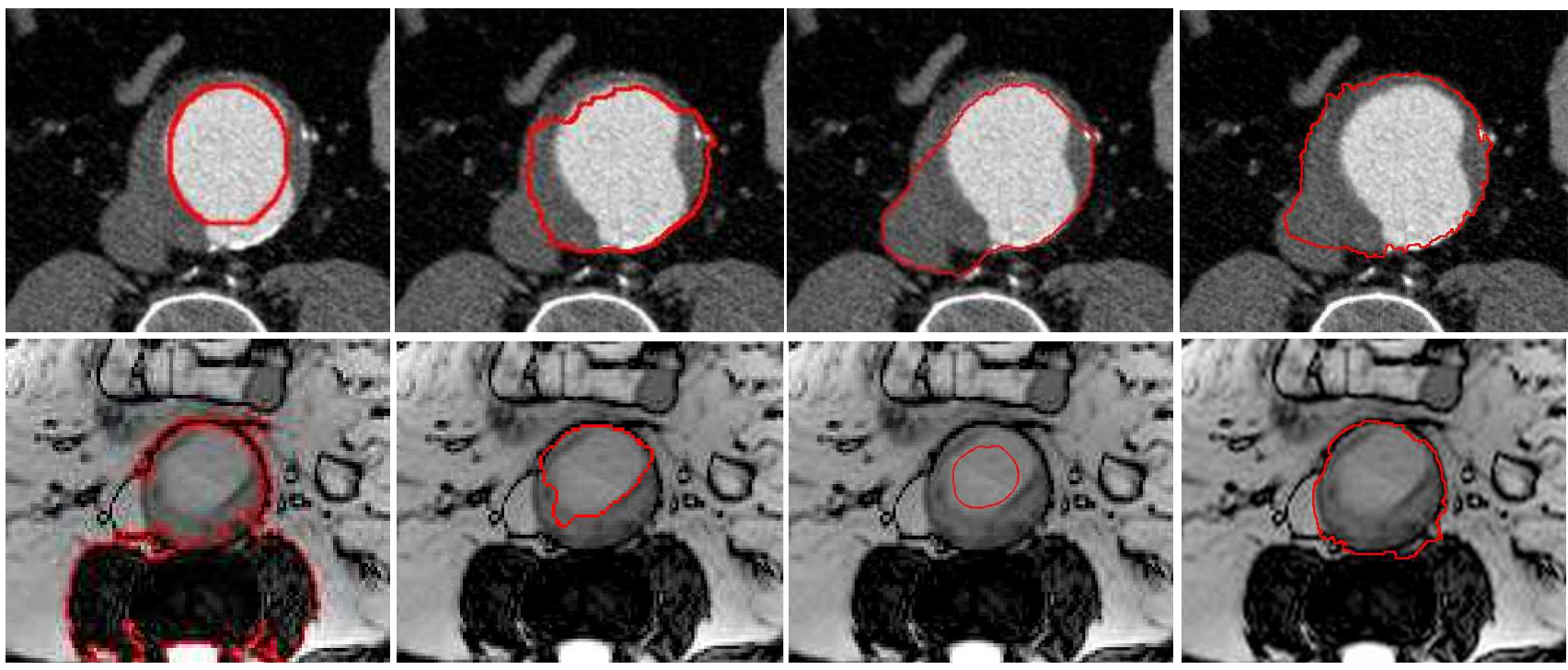

Figure 2. Different segmentation results obtained on a CT and an MR image. These results are typical of the type of issues one has to deal with. (First row) Results obtained with a snake with an ovoid shape prior [9], graph-cut [13], grow cut [15], graph cut with star shape prior [38] (second row) flood fill [12], iterative graph cut, level set [7], and graph cut with star shape prior [38]. Let us mention that the initialization step is similar for all of these methods. 

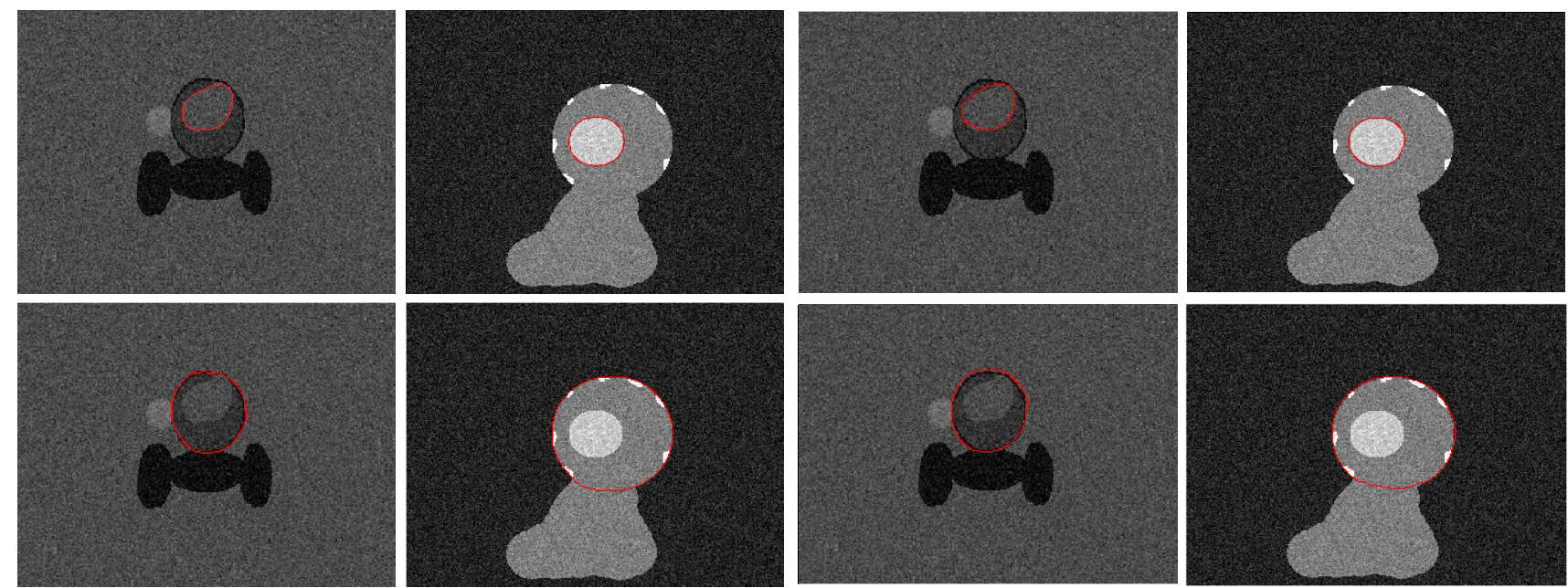

Figure 3. Synthetic version of an MR image (first and third column) and a CT-scan image (second and fourth column). The MR image contains Rician noise with standard deviation of 15 while the CT-scan image contains Gaussian noise with standard deviation of 20. The four left most images were hand segmented by an expert while the four right most images have been segmented with our method.
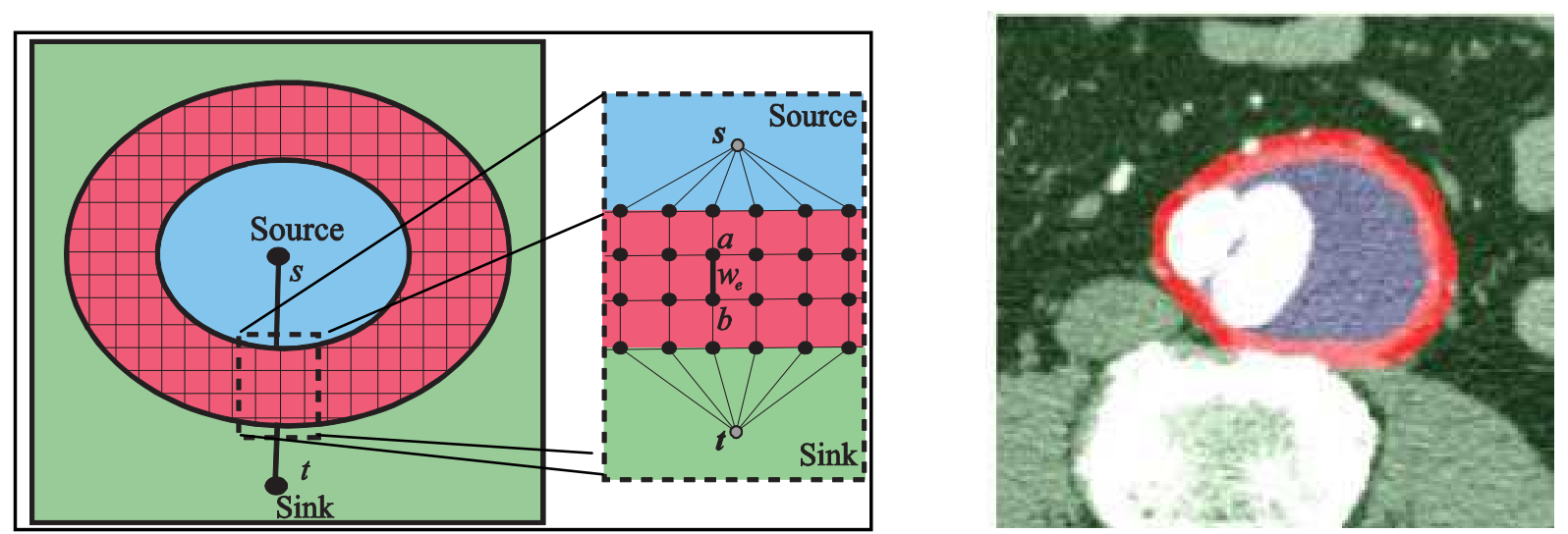

Figure 4. (left) Schematic representation and (right) snapshot of an initial contour entered by the user. Pixels inside the blue area are connected to the source with an infinite capacity and those in the green area are also connected to the sink with an infinite capacity. The pixels located in the red section are connected to their 6 neighbours ( 4 in the same image, 1 in the image above, and 1 in the image below). The weight $w_{e}$ of these 6 edges is determined by equation 3 .

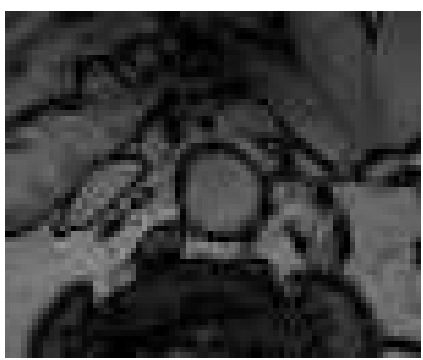

Input image

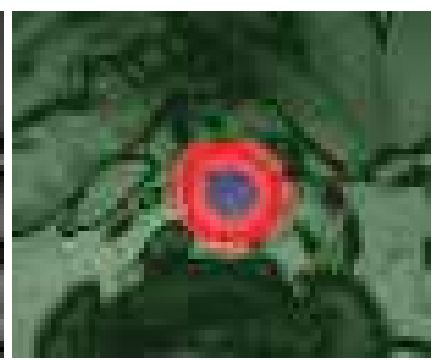

User selection

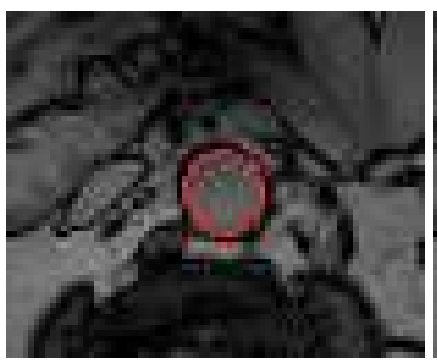

lumen segmentation

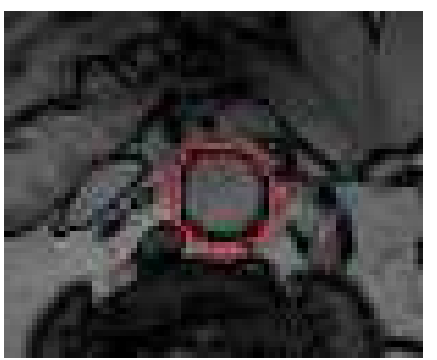

Wall segmentation

Figure 5. Given an input image and a user selection, the last two images illustrate the effect of equation (4) and (5). 


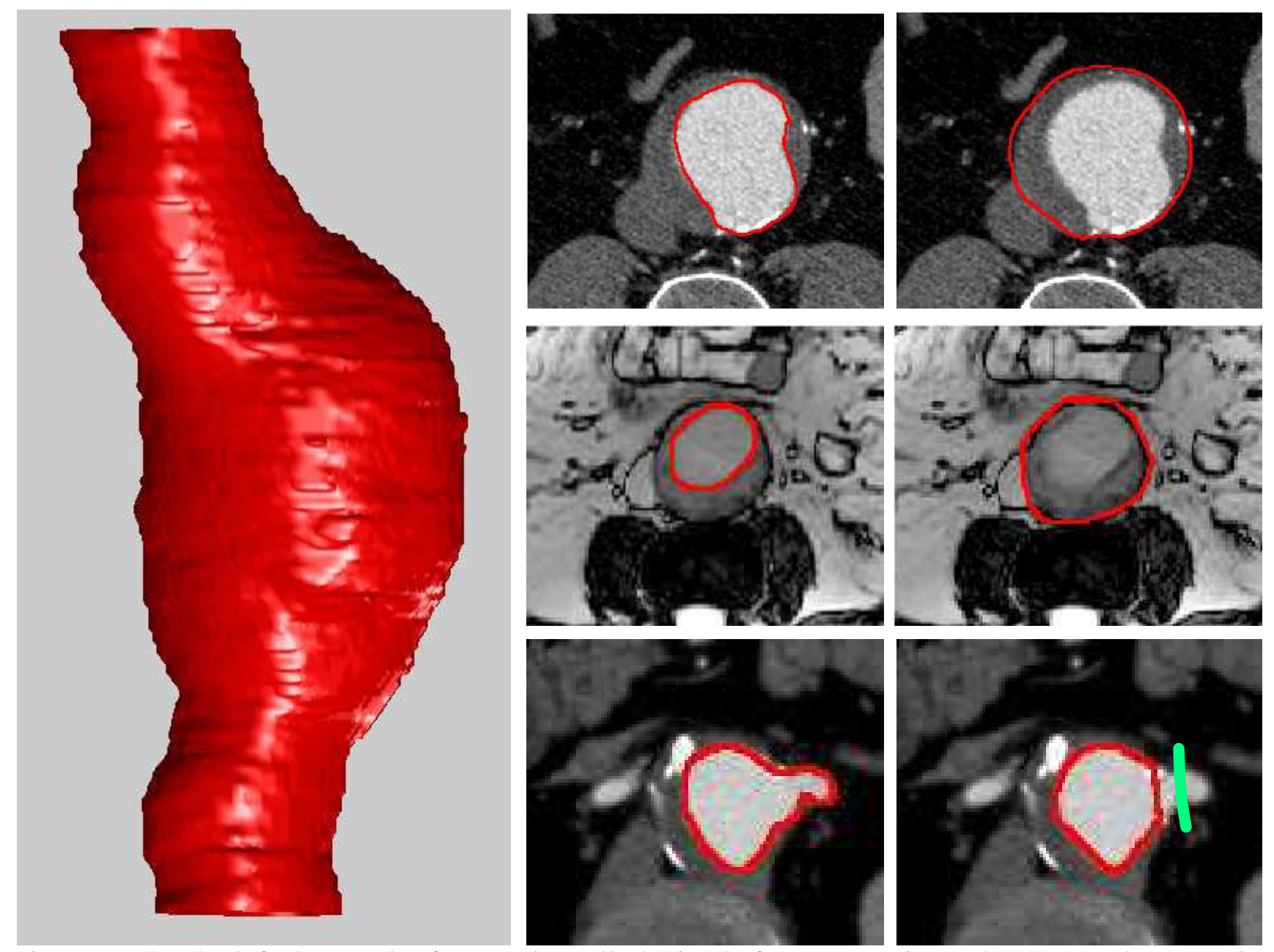

Figure 6. On the left, 3D result of an aortic wall obtained after segmenting a CT-Scan volume made of 129 images. On the right, result obtained with our method for the lumen and the wall in a CT-scan and an MRI. The last two images illustrate the touch up tool. 

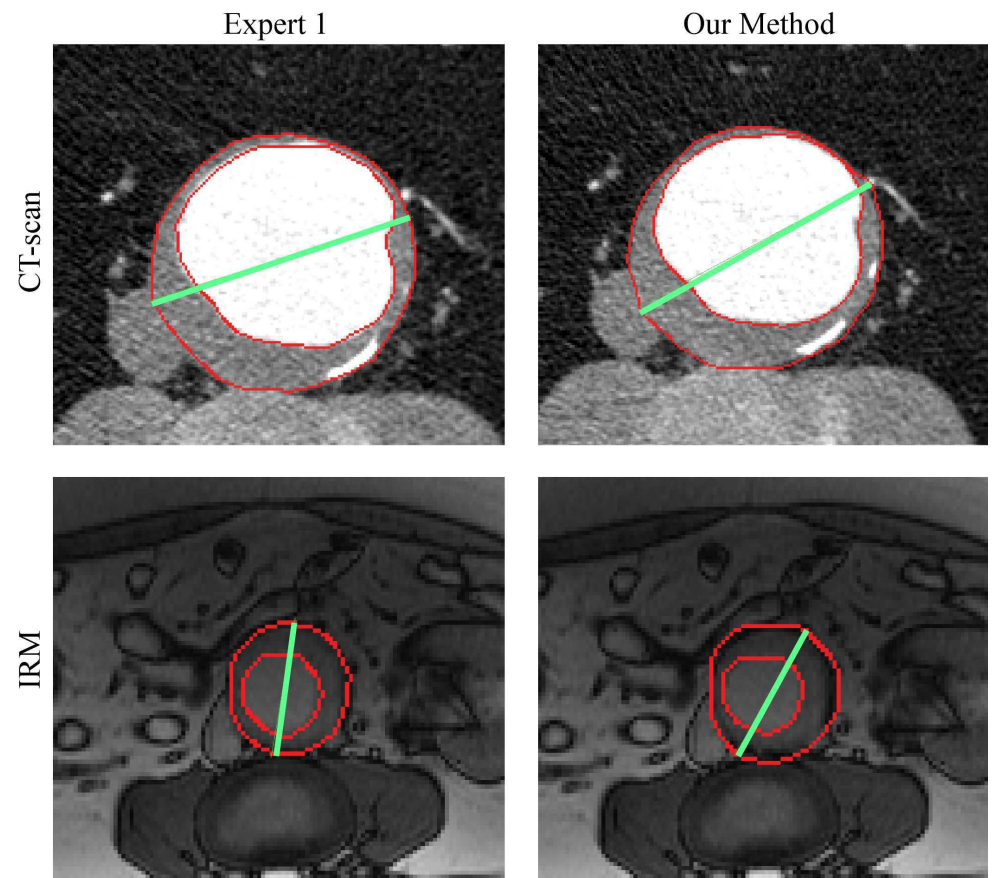

Figure 7. Typical results produced by an expert and by our method on CT-scan and MRI data. The difference is barely noticeable by an human eye, especially for the CT-scan. The green line corresponds to the maximum diameter.

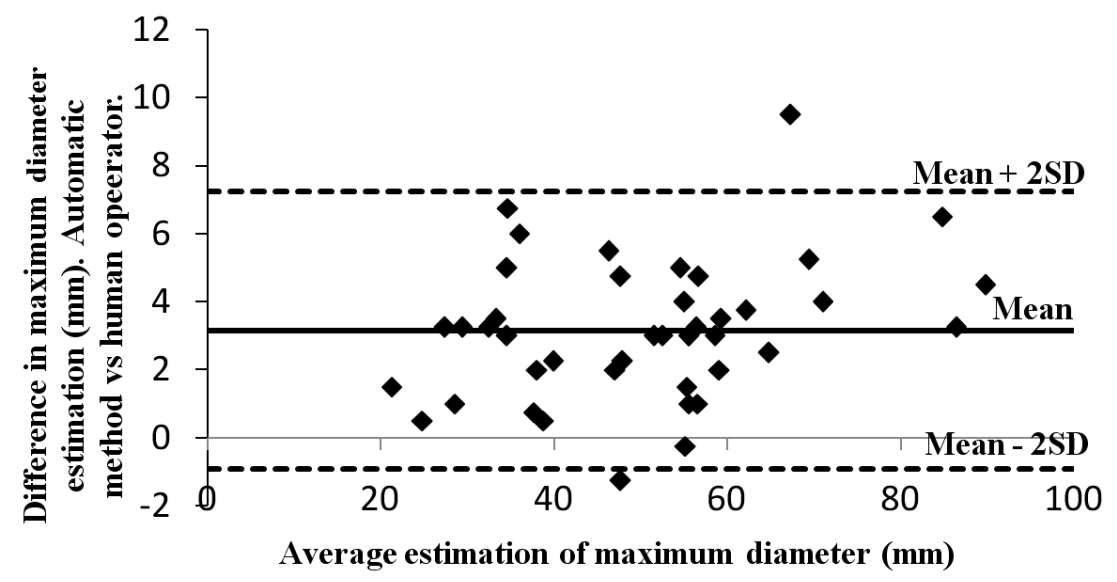

Figure 8 : Comparison between automatic and manual processing for the evaluation of the maximum diameter with a Bland-Altman plot (mean $=3.2 \mathrm{~mm}$; standard deviation $(\mathrm{SD})=2 \mathrm{~mm}$ ) 
Table 1 Acquisition parameters and study population

\begin{tabular}{|c|c|c|}
\hline & MRI & $\overline{\mathrm{CT}}$ \\
\hline System & $\begin{array}{c}\text { 3T whole body Imager (10 patients) } \\
\text { (Trio TIM. Siemens Medical Solution, } \\
\text { Erlangen, Germany) } \\
\\
1.5 \text { T whole body imager } \\
\text { (4 patients and } 6 \text { healthy subjects) } \\
\text { (Avanto TIM. Siemens Medical } \\
\text { Solution, Erlangen, Germany) }\end{array}$ & $\begin{array}{c}\text { Spiral CT scanner with intravenous } \\
\text { injection of contrast material } \\
\text { (LightSpeed Ultra, } \\
\text { GE Medical Systems, USA) }\end{array}$ \\
\hline $\begin{array}{l}\text { Patients with } \\
\text { AAA }\end{array}$ & $\begin{array}{l}14 \text { (13 men, } 1 \text { woman }) \\
70 \pm 10 \text { years }\end{array}$ & $\begin{array}{c}21 \text { men } \\
70 \pm 11 \text { years }\end{array}$ \\
\hline $\begin{array}{l}\text { Patients without } \\
\text { AAA }\end{array}$ & $\begin{array}{c}6(4 \text { men, } 2 \text { women }) \\
63 \pm 14 \text { years }\end{array}$ & $\begin{array}{c}3 \text { (2 men, } 1 \text { woman }) \\
70 \pm 2 \text { years }\end{array}$ \\
\hline Image size & $256 \times 176$ & $512 \times 512$ \\
\hline Spatial resolution & $\begin{array}{c}1.12 \mathrm{~mm} \text { to } 1.40 \mathrm{~mm} \text { per pixel } \\
\text { according to the subject }\end{array}$ & $\begin{array}{c}0.47 \mathrm{~mm} \text { to } 0.98 \mathrm{~mm} \text { per pixel } \\
\text { according to the subject }\end{array}$ \\
\hline Slice thickness & $5 \mathrm{~mm}$ & $\begin{array}{c}1 \mathrm{~mm} \text { to } 8 \mathrm{~mm} \text { per pixel according to } \\
\text { the subject }\end{array}$ \\
\hline
\end{tabular}

Table 2 Volume overlap (\%) for diseased aortas

\begin{tabular}{|l|c|c|c|c|c|c|c|c|c|c|}
\hline & \multicolumn{2}{|c|}{ Expert 1 } & \multicolumn{2}{c|}{ Expert 2 } & \multicolumn{2}{c|}{ Expert 3 } & \multicolumn{2}{c|}{ Expert 4 } & \multicolumn{2}{c|}{ Our method } \\
\hline & $\mu$ & $\sigma$ & $\mu$ & $\sigma$ & $\mu$ & $\sigma$ & $\mu$ & $\sigma$ & $\mu$ & $\sigma$ \\
\hline Lumen, MRI & 86.26 & 4.44 & 85.85 & 5.35 & 85.83 & 5.16 & 83.86 & 5.78 & 83.07 & 5.25 \\
\hline Wall, MRI & 88.57 & 5.67 & 87.93 & 6.13 & 88.25 & 5.61 & 83.72 & 5.75 & 85.24 & 6.09 \\
\hline Lumen, CTscan & 90.47 & 4.83 & 90.83 & 4.43 & 90.32 & 4.86 & 87.01 & 6.73 & 87.69 & 6.83 \\
\hline Wall, CTscan & 92.89 & 3.35 & 91.29 & 4.01 & 92.60 & 3.36 & 92.23 & 3.55 & 90.93 & 4.90 \\
\hline
\end{tabular}

Table 3 Hausdorff distance ( $\mathrm{mm}$ ) for diseased aortas

\begin{tabular}{|l|c|c|c|c|c|c|c|c|c|c|}
\hline & \multicolumn{2}{|c|}{ Expert 1 } & \multicolumn{2}{c|}{ Expert 2 } & \multicolumn{2}{c|}{ Expert 3 } & \multicolumn{2}{c|}{ Expert 4 } & \multicolumn{2}{c|}{ Our method } \\
\hline & $\mu$ & $\sigma$ & $\mu$ & $\sigma$ & $\mu$ & $\sigma$ & $\mu$ & $\sigma$ & $\mu$ & $\sigma$ \\
\hline Lumen, MRI & 2.43 & 0.95 & 2.64 & 1.16 & 2.54 & 1.05 & 2.79 & 1.15 & 2.92 & 1.10 \\
\hline Wall, MRI & 2.84 & 1.24 & 2.97 & 1.53 & 2.84 & 1.30 & 3.34 & 1.17 & 3.52 & 1.50 \\
\hline Lumen, CTscan & 2.13 & 0.92 & 2.17 & 1.05 & 2.37 & 1.10 & 2.61 & 1.17 & 2.68 & 1.25 \\
\hline Wall, CTscan & 2.33 & 1.17 & 2.70 & 1.39 & 2.51 & 1.36 & 2.57 & 1.54 & 3.09 & 1.81 \\
\hline
\end{tabular}

Table 4 Maximum diameter distance $(\mathrm{mm})$ for diseased aortas

\begin{tabular}{|l|c|c|c|c|c|c|c|c|c|c|}
\hline & \multicolumn{2}{|c|}{ Expert 1 } & \multicolumn{2}{c|}{ Expert 2 } & \multicolumn{2}{c|}{ Expert 3 } & \multicolumn{2}{|c|}{ Expert 4 } & \multicolumn{2}{|c|}{ Our method } \\
\hline & $\mu$ & $\sigma$ & $\mu$ & $\sigma$ & $\mu$ & $\sigma$ & $\mu$ & $\sigma$ & $\mu$ & $\sigma$ \\
\hline MRI & 2.55 & 1.70 & 2.26 & 1.50 & 2.71 & 1.83 & 3.16 & 2.03 & 3.39 & 2.08 \\
\hline CTscan & 2.04 & 1.76 & 2.01 & 1.47 & 2.12 & 1.47 & 2.96 & 2.35 & 2.86 & 1.77 \\
\hline
\end{tabular}


Table 5 Volume overlap (\%) for healthy aortas

\begin{tabular}{|l|c|c|c|c|c|c|c|c|c|c|}
\hline & \multicolumn{2}{|c|}{ Expert 1 } & \multicolumn{2}{c|}{ Expert 2 } & \multicolumn{2}{c|}{ Expert 3 } & \multicolumn{2}{c|}{ Expert 4 } & \multicolumn{2}{c|}{ Our method } \\
\hline & $\mu$ & $\sigma$ & $\mu$ & $\sigma$ & $\mu$ & $\sigma$ & $\mu$ & $\sigma$ & $\mu$ & $\sigma$ \\
\hline Lumen, MRI & 89.70 & 5.61 & 89.90 & 6.86 & 89.50 & 5.69 & 88.13 & 6.41 & 86.60 & 5.95 \\
\hline Wall, MRI & 88.94 & 7.01 & 88.64 & 7.50 & 88.67 & 5.35 & 82.68 & 7.42 & 81.64 & 7.03 \\
\hline Lumen, CTscan & 88.84 & 4.25 & 89.23 & 4.99 & 88.45 & 4.94 & 84.85 & 6.13 & 86.52 & 6.37 \\
\hline Wall, CTscan & 89.40 & 4.53 & 86.56 & 6.03 & 88.63 & 4.88 & 88.65 & 4.97 & 83.85 & 6.13 \\
\hline
\end{tabular}

Table 6 Hausdorff distance $(\mathrm{mm})$ for healthy aortas

\begin{tabular}{|l|c|c|c|c|c|c|c|c|c|c|}
\hline & \multicolumn{2}{|c|}{ Expert 1 } & \multicolumn{2}{c|}{ Expert 2 } & \multicolumn{2}{c|}{ Expert 3 } & \multicolumn{2}{c|}{ Expert 4 } & \multicolumn{2}{c|}{ Our method } \\
\hline & $\mu$ & $\sigma$ & $\mu$ & $\sigma$ & $\mu$ & $\sigma$ & $\mu$ & $\sigma$ & $\mu$ & $\sigma$ \\
\hline Lumen, MRI & 1.87 & 2.04 & 1.97 & 2.39 & 1.90 & 1.91 & 2.41 & 3.00 & 2.25 & 1.95 \\
\hline Wall, MRI & 2.27 & 1.46 & 2.36 & 1.90 & 2.42 & 1.60 & 3.21 & 2.31 & 3.45 & 1.59 \\
\hline Lumen, CTscan & 1.41 & 0.38 & 1.39 & 0.42 & 1.49 & 0.43 & 1.70 & 0.39 & 1.61 & 0.52 \\
\hline Wall, CTscan & 1.69 & 0.56 & 2.00 & 0.69 & 1.82 & 0.62 & 1.83 & 0.62 & 2.31 & 0.74 \\
\hline
\end{tabular}

Table 7 Maximum diameter distance $(\mathrm{mm})$ for healthy aortas

\begin{tabular}{|l|c|c|c|c|c|c|c|c|c|c|}
\hline & \multicolumn{2}{|c|}{ Expert 1} & \multicolumn{2}{c|}{ Expert 2} & \multicolumn{2}{c|}{ Expert 3 } & \multicolumn{2}{c|}{ Expert 4 } & \multicolumn{2}{c|}{ Our method } \\
\hline & $\mu$ & $\sigma$ & $\mu$ & $\sigma$ & $\mu$ & $\sigma$ & $\mu$ & $\sigma$ & $\mu$ & $\sigma$ \\
\hline MRI & 2.65 & 1.63 & 2.61 & 2.25 & 2.58 & 2.05 & 2.64 & 2.67 & 4.86 & 2.01 \\
\hline CTscan & 1.93 & 1.40 & 1.35 & 1.12 & 1.53 & 0.91 & 3.05 & 1.24 & 1.96 & 1.54 \\
\hline
\end{tabular}

Table 8 Volume overlap (\%) for synthetic images

\begin{tabular}{|l|c|c|c|c|c|c|}
\hline & \multicolumn{3}{|c|}{ Expert 1 } & \multicolumn{3}{c|}{ Our Method } \\
\hline \multicolumn{1}{|c|}{ Noise Level } & $\boldsymbol{\sigma}=\mathbf{1}$ & $\boldsymbol{\sigma = 1 0}$ & $\boldsymbol{\sigma = 1 5}$ & $\boldsymbol{\sigma = 1}$ & $\boldsymbol{\sigma = 1 0}$ & $\boldsymbol{\sigma = 1 5}$ \\
\hline Lumen, MRI & 97.82 & 96.74 & 95.37 & 100 & 92.05 & 91.55 \\
\hline Wall, MRI & 96.51 & 96.94 & 95.83 & 96.46 & 95.37 & 93.26 \\
\hline \multicolumn{1}{|c|}{ Noise Level } & $\boldsymbol{\sigma}=\mathbf{1}$ & $\boldsymbol{\sigma}=\mathbf{1 0}$ & $\boldsymbol{\sigma = 2 0}$ & $\boldsymbol{\sigma = 1}$ & $\boldsymbol{\sigma}=\mathbf{1 0}$ & $\boldsymbol{\sigma}=\mathbf{2 0}$ \\
\hline Lumen, CTscan & 99.16 & 99.51 & 98.74 & 99.16 & 94.91 & 95.62 \\
\hline Wall, CTscan & 98.47 & 98.64 & 98.67 & 96.03 & 95.69 & 96.44 \\
\hline
\end{tabular}

Table 9 Hausdorff distance $(\mathrm{mm})$ for synthetic images

\begin{tabular}{|l|c|c|c|c|c|c|}
\hline & \multicolumn{3}{|c|}{ Expert 1 } & \multicolumn{3}{c|}{ Our Method } \\
\hline \multicolumn{1}{|c|}{ Noise Level } & $\boldsymbol{\sigma}=\mathbf{1}$ & $\boldsymbol{\sigma = 1 0}$ & $\boldsymbol{\sigma = 1 5}$ & $\boldsymbol{\sigma = 1}$ & $\boldsymbol{\sigma = 1 0}$ & $\boldsymbol{\sigma = 1 5}$ \\
\hline Lumen, MRI & 1.00 & 1.00 & 1.00 & 1.00 & 2.83 & 2.24 \\
\hline Wall, MRI & 1.41 & 1.41 & 2.00 & 2.00 & 2.83 & 2.24 \\
\hline \multicolumn{1}{|c|}{ Noise Level } & $\boldsymbol{\sigma}=\mathbf{1}$ & $\boldsymbol{\sigma = 1 0}$ & $\boldsymbol{\sigma}=\mathbf{2 0}$ & $\boldsymbol{\sigma}=\mathbf{1}$ & $\boldsymbol{\sigma}=\mathbf{1 0}$ & $\boldsymbol{\sigma = 2 0}$ \\
\hline Lumen, CTscan & 1.00 & 1.00 & 1.00 & 1.00 & 2.24 & 1.41 \\
\hline Wall, CTscan & 2.00 & 2.00 & 1.41 & 4.47 & 4.00 & 3.00 \\
\hline
\end{tabular}


Table 10 Maximum diameter distance (mm) for synthetic images

\begin{tabular}{|c|c|c|c|c|c|c|}
\hline & \multicolumn{3}{|c|}{ Expert 1} & \multicolumn{3}{c|}{ Our Method } \\
\hline Noise Level & $\boldsymbol{\sigma}=\mathbf{1}$ & $\boldsymbol{\sigma = 1 0}$ & $\boldsymbol{\sigma = 1 5}$ & $\boldsymbol{\sigma = 1}$ & $\boldsymbol{\sigma = \mathbf { 1 0 }}$ & $\boldsymbol{\sigma = 1 5}$ \\
\hline MRI & 1.00 & 1.38 & 1.67 & 1.01 & 1.03 & 1.59 \\
\hline Noise Level & $\boldsymbol{\sigma}=\mathbf{1}$ & $\boldsymbol{\sigma}=\mathbf{1 0}$ & $\boldsymbol{\sigma}=\mathbf{2 0}$ & $\boldsymbol{\sigma}=\mathbf{1}$ & $\boldsymbol{\sigma}=\mathbf{1 0}$ & $\boldsymbol{\sigma = 2 0}$ \\
\hline CTscan & 1.23 & 0.39 & 0.55 & 0.84 & 0.84 & 1.45 \\
\hline
\end{tabular}

\section{BIOBLIOGRAPHY}

[1] Isselbacher EM. Thoracic and abdominal aortic aneurysms. Circulation 2005; 111(6):816-828.

[2] Nevitt MP, Ballard DJ, Hallett JW. Prognosis of abdominal aortic aneurysms: a population-based study. N Engl J Med 1989; 321:1009-1014.

[3] Zarins C, Crabtree T, Bloch D, Arko F, Ouriel K, White R. Endovascular aneurysm repair at 5 years: Does aneurysm diameter predict outcome? J Vasc Surg 2006; 44: 920-929.

[4] Al-Omran M, Verma S, Lindsay TF, Weisel RD, Sternbach Y. Clinical decision making for endovascular repair of abdominal aortic aneurysm, Circulation 2004; 110(23): 517-523.

[5] Greenhalgh RM, Powell JT. Endovascular repair of abdominal aortic aneurysm. N Engl J Med 2008; 358:494-501.

[6] Cremers D, Schnörr C, Weickert J. Diffusion Snakes using Statistical Shape knowledge. Algeb Frames Percep Action Cycle 2000; 1888:164-174.

[7] Chan TF, Vese LA. Active Contours Without Edges. In: Inter Conf Image Proc; 2001; 10:266277.

[8] Caselles V, Kimmel R, Sapiro G. Geodesic active contours. Inter J Compu Vision 1997; 22:6179.

[9] Lee ET, Pan YJ, Chu P. An Algorithm for Region Filling using Two-Dimensional Grammars. Inter Jour Info Secu 1987; 2(3): 255-263.

[10] Mansouri AR, Mitiche A, Vazquez C. Multiregion competition: a level set extension of region competition to multiple region image partitioning. Compu. Vision Image Under 2006; 101:137-150

[11] Osher S, Fedkiw R. Level Set Methods and Dynamic Implicit Surfaces. Springer 153; 2003.

[12] Xu N, Ahuja N, Bansal R. Object Segmentation using Graph Cuts based Active Contours. Comp Vision Image Under 2007; 107: 210-224.

[13] Boykov Y, Jolly MP. Interactive Graph Cuts for Optimal Boundary and Region Segmentation of Objects in N-D Images. In: proc of ICCV; 2001; 1: 105-112. 
[14] Boykov Y, Jolly MP. Interactive Organ Segmentation using Graph Cuts. In: Medical Image Comp Compu Assi Inter; 2000: 276-286.

[15] Vezhnevets V, Konouchine V. GrowCut - Interactive Multi-Label N-D Image Segmentation by Cellular Automata. In: Graphicon: 2005.

[16] Bieniek A, Moga A. An efficient watershed algorithm based on connected components. Pattern Recognition 2000; 33: 907-916.

[17] Freiman M, Broide N, Natanzon M, Nammer E, Shilon O, Weizman L, Joskowicz L, Sosna J. Vessels-Cut: A Graph Based Approach to Patient-Specific Carotid Arteries Modeling. In: 3D Physio Human Lecture Notes Compu Science; 2009; 5903:1-12.

[18] Zhao F, Zhang H, Wahle A, Scholz TD, Sonka M. Automated 4D Segmentation of Aortic Magnetic Resonance Images. In: Brit Mach Vision Conf; 2006; 1: 247-256.

[19] Kovacs T, Cattin P, Alkadhi H, Wildermuth S, Székely G. Automatic Segmentation of the Vessel Lumen from 3D CTA Images of Aortic Dissection. Bildverarbeitung für die Medizin 2006: 161-165.

[20] Chul Kim H, Park SW, Nam KW, Choi H, Choi EJ, Jin S, Kim MG, Sun K. Determination of accurate stent graft configuration in abdominal aortic aneurysm using computed tomography: a preliminary study. Clinical Imaging 2010; 34: 255-262

[21] Jodoin PM, Lalande A, Voisin Y, Bouchot O, Steinmetz E. Markovian Method for 2D, 3D and 4D Segmentation of MRI. In: Inter Conf Image Proc; 2008: 3012-3015.

[22] Couprie C, Grady L, Najman L, Talbot H. Power Watersheds: A Unifying Graph-Based Optimization Framework, IEEE trans on PAMI 2010; 33(7): 1384-1399.

[23] Blake A, Rother C, Brown M, Perez $\mathrm{P}$, Torr $\mathrm{P}$. Interactive image segmentation using an adaptive GMMRF model. In: Proc of ECCV; 2004: 428-44.

[24] Miranda P, Falcao AX, Udupa JK. Cloud Bank: A Multiple Clouds Model and its use in MR Brain Image Segmentation. In: Proc. of the IEEE ISBI; 2009, 506-509.

[25] Leventon M, Grimson WEL, Faugeras O. Statistical Shape Influence in Geodesic Active Contours. In: proc of the IEEE CVPR; 2000; 1: 316-323

[26] Tsai A, Yezzi AJ, Wells W, Tempany C, Tucker D, Fan A, Grimson W, Willsky A. A shapebased approach to the segmentation of medical imagery using level sets, IEEE trans Medical Imaging 2003; 22(2): 137-154.

[27] Chan T, Zhu W. Level Set Based Shape Prior Segmentation. In: proc. of CVPR; 2005:11641170.

[28] Cremers D, Tischhauser F, Weickert J, Schnorr C. Diffusion snakes: introducing statistical shape knowledge into the mumford-shah functional. Int. J. of Computer Vision 2002; 50(3): 295-313. 
[29] Wu XQ, Shah SK. Level Set with Embedded Conditional Random Fields and Shape Priors for Segmentation of Overlapping Objects. In: proc of ACCV; 2010; 230-241.

[30] Leventon ME, Grimson WEL, Faugeras O. Statistical Shape Influence in Geodesic Active Contours. In: Comp Vision Patt Recogn; 2000; 1:316-322.

[31] Chen Y, Thiruvenkadam S., Tagare H, Huang F, Wilson D, Geiser E. On the Incorporation of shape priors into geometric active contours. In: Proc of IEEE VLSM; 2001: 145-152.

[32] Slabaugh G, Unal G. Graph cuts segmentation using an elliptical shape prior. In: Proc of ICIP; 2005: 1222-1225

[33] Das P, Veksler O, Zavadsky S, Boykov Y. Semiautomatic segmentation with compact shapre prior. In: Prof of CRV; 2006: 28-36.

[34] Freedman D, Zhang T. Interactive graph cut based segmentation with shape priors. In: Proc of CVPR; 2005; I: 755-762.

[35] Vicente S, Kolmogorov V, Rother C. Graph cut based image segmentation with connectivity priors. In: proc of CVPR; 2008.

[36] Funka-Lea G, Boykov Y, Florin C, Jolly M, Moreau-Gobard R, Ramaraj R, Rinck D. Automatic heart isolation for ct coronary visualization using graph-cuts. In: ISBI; 2006: 614-617.

[37] Kumar M, Torr P, Zisserman A. Obj cut. In: proc. of CVPR; 2005: 18-25.

[38] Veksler O. Star Shape Prior for Graph-Cut Image Segmentation. In proc of ECCV; 2008. 454467.

[39] Freiman M, Esses S, Joskowicz L, Sosna J. An Iterative Model-Constraint Graph-cut Algorithm for Abdominal Aortic Aneurysm Thrombus Segmentation. In: Inter Sympo Biome Imaging; 2010: $672-675$.

[40] Deng X, Du G. Editorial: 3D Segmentation in the Clinic: A Grand Challenge II - Liver Tumor Segmentation. In: MICCAI; 2008;1-4.

[41] Rote G. Computing the minimum Hausdorff distance between two point sets on a line under translation". Info Proc Letters 1991; 38:123-127

[42] Gudbjartsson H, Patz S. The Rician Distribution of Noisy MRI Data, Magn Reson Med 1995; 34(6): 910-914

[43] Collins T. Graph Cut Matching In Computer Vision. University of Edinburgh; 2004.

[44] Ford LR, Fulkerson DR. Maximal Flow Through a Network. Cana J Math 1956; 8:399-404.

[45] Stoer M, Wagner F. A Simple Min-Cut Algorithm. J Asso Compu Mach 1997; 44: 585-591.

[46] BoykovY, Veksler O, Zabih R. Fast Approximate Energy Minimization via Graph Cuts. IEEE trans on PAMI 2011; 23:1222-1239 
[47] Knorr EM, Ng RT. Finding Intentional Knowledge of Distance-based Outliers. In: VLDB'99; $1999 ; 1: 12$.

[48] Kim SJ. The Metrically Trimmed Mean as a Robust Estimator of Location. Annals Statistics 1992; 20:1534-1547.

[49] Bland JM, Altman DG. Statistical methods for assessing agreement between two methods of clinical measurement. Lancet 1986; $8: 307-317$. 